\title{
2-GENERATOR GROUPS AND PARABOLIC CLASS NUMBERS
}

\author{
MORRIS NEWMAN
}

\begin{abstract}
It is shown that if $x, y$ are generators of the finite group $G$ such that $x^{p}=y^{q}=(x y)^{n}=1$, where $p, q, n$ are integers $>1,(p, q)=1$, and $x y$ is of true order $n$, then the order $\mu=n t$ of $G$ satisfies $n \leqq p q t^{p}$. This result is used to show that if $F$ is a Fuchsian group of genus 0 generated by 2 elliptic elements of coprime order and with 1 parabolic class, then $F$ possesses only finitely many normal subgroups having a given number of parabolic classes.
\end{abstract}

Introduction. Let $\Gamma=L F(2, Z)$ be the classical modular group, so that $\Gamma$ is the free product of the cyclic group $\{T\}$ of order 2 and the cyclic group $\{U\}$ of order 3 , where $T=\left(\begin{array}{cc}0 & 1 \\ -1 & 0\end{array}\right), U=\left(\begin{array}{cc}0 & -1 \\ 1 & 1\end{array}\right)$, and $S=T U=\left(\begin{array}{ll}1 & 1 \\ 0 & 1\end{array}\right)$ is parabolic. Let $G$ be a normal subgroup of $\Gamma$ of index $\mu$ and level $n$ (the least positive integer such that $S^{n} \in G$ ). Then if $t$ is the number of parabolic classes of $G, t=\mu / n$. In [3] the conjecture was made that there are only finitely many normal subgroups of $\Gamma$ having a given number $t$ of parabolic classes. This conjecture was proved by $\mathrm{L}$. Greenberg in his paper [2], who showed that $n \leqq 6 t^{3}$, so that $\mu \leqq 6 t^{4}$.

In connection with his work on automorphisms of closed Riemann surfaces, R. Accola [1] proved a number of group-theoretic lemmas about finite groups generated by 2 elements one of which is of period 2 . A consequence of his work is the improvement of the inequality above to $n \leqq 6 t^{2}$ always, and $n \leqq t^{2}$ if $\Gamma / G$ is not abelian.

In this paper we apply Accola's method with some modifications to the more general situation of any finite group $G$ generated by 2 elements, whose orders are assumed relatively prime for our purpose. We obtain a number of inequalities connecting the order of $G$ with the orders of the elements and their products. The results are used to show that Fuchsian groups of genus 0 with two elliptic generators of coprime order and with one parabolic class have the same sort of property as $\Gamma$ with respect to the parabolic class number.

The author is indebted to the referee for correcting an error in the original version of the paper.

Received by the editors November 2, 1970.

AMS 1970 subject classifications. Primary 20H10, 20D99; Secondary 10D05.

Key words and phrases. Parabolic classes, Fuchsian groups, 2-generator groups. 
The group-theoretical results. We have the following lemma:

Lemma 1. Suppose that the group $G$ possesses a central normal subgroup $C$ of index $n$. Let $m$ be any multiple of $n$. Then the mapping $g \rightarrow g^{m}, g \in G$, is a homomorphism.

ProOF. The mapping $g \rightarrow g^{n}$ is just the transfer homomorphism of $G$ into $C$, so the result follows (see [4, Chapter V]).

Now let $p, q, n$ be integers $>1$ such that $(p, q)=1$. Let $G=\{x, y\}$ be a group of order $\mu$ such that $x^{p}=y^{q}=(x y)^{n}=1$, and $z=x y$ is of true order $n$. Since $n \mid \mu$, we may write $\mu=n t$.

We are interested in inequalities involving $\mu, n, t$. We shall prove

THEOREM 1. The quantities $\mu, n, t$ satisfy the following inequalities:

$$
\begin{aligned}
& n \leqq p q t^{p}, \\
& \mu \leqq p q t^{p+1}, \\
& \mu \geqq(p q)^{-1 / p} n^{1+1 / p} .
\end{aligned}
$$

Proof. The inequalities are entirely equivalent, and it is sufficient to prove any one of them. Put $C=\bigcap_{i=0}^{p-1}\left\{x^{i} z x^{-i}\right\}$. Then clearly, $C$ is normalized by $z$ (since $C$ is a subgroup of the cyclic group $\{z\}$ ), and also, $C$ is normalized by $x$. Thus $C$ is in fact a normal subgroup of $G$, since $x$ and $z$ generate $G$. Also for some divisor $k$ of $n, C=\left\{z^{k}\right\}$, so that $C$ is of order $n / k$ and index $k t$. Furthermore, Poincaré's index inequality implies that

$$
(G: C) \leqq \prod_{i=0}^{p-1}\left(G:\left\{x^{i} z x^{-i}\right\}\right), \quad k t \leqq t^{p}, \quad k \leqq t^{p-1}
$$

We now remark that the elements $x^{i} z x^{-i}, 0 \leqq i \leqq p-1$, generate $G$. For if

$$
H=\left\{z, x z x^{-1}, \cdots, x^{p-1} z x^{-(p-1)}\right\},
$$

then $z$ normalizes $H$ (since $z \in H$ ) and $x$ normalizes $H$, so that $H$ is a normal subgroup of $G$. Since $x y \in H$, we have that, in $G / H, x^{p}=y^{q}=1$, $x y=1$, so that $x=y^{-1}, x^{q}=y^{-q}=1$. Since $(p, q)=1, x=1$; and thus also $y=1$. It follows that $G / H$ is trivial, and so $G=H$.

Now $x^{-i} z^{k} x^{i}=z^{k l_{i}}, 0 \leqq i \leqq p-1$, since $C$ is a cyclic normal subgroup of $G$ generated by $z^{k}$. Hence

$$
\begin{aligned}
\left(x^{i} z x^{-i}\right)^{-1} z^{k}\left(x^{i} z x^{-i}\right) & =x^{i} z^{-1} x^{-i} z^{k} x^{i} z x^{-1}=x^{i} z^{-1} z^{k l_{i}} z x^{-1} \\
& =x^{i} z^{k l_{i}} x^{-1}=z^{k} .
\end{aligned}
$$

It follows that $C$ is a central normal subgroup of $G$. By Lemma 1, the mapping $\varphi: g \rightarrow g^{p q k t}, g \in G$, is a homomorphism. Since $\varphi: x \rightarrow 1$ and $\varphi: y \rightarrow 1, \varphi: g \rightarrow 1$ for all $g \in G$. In particular, $z^{p q k t}=1$. It follows that 
$p q k t \equiv 0 \bmod n$, and hence $n \leqq p q k t \leqq p q t^{p}$, which is just (1). This completes the proof.

The restriction that $(p, q)=1$ is essential, as may be seen from the dihedral group, for example.

An application. As a consequence of Theorem 1, we prove

THEOREM 2. Let $F$ be the Fuchsian group with presentation

$$
E_{1}^{p}=E_{2}^{a}=1, \quad E_{1} E_{2} P=1,
$$

where $P$ is parabolic and $p, q$ are relatively prime integers $>1$. Then $F$ has only finitely many normal subgroups of finite index having a given number $t$ of parabolic classes.

Proof. Let $G$ be a normal subgroup of $F$ of index $\mu$ having $t$ parabolic classes. Then if $P$ is of exponent $n$ modulo $G, t=\mu / n$. In the group $F / G$, which is of order $\mu=n t$, we have

$$
E_{1}^{p}=E_{2}^{q}=\left(E_{1} E_{2}\right)^{n}=1,
$$

and $E_{1} E_{2}$ is of true order $n$. By the previous theorem, we have $\mu \leqq p q t^{p+1}$. Since the index is bounded, and since $F$ is finitely generated, there are only finitely many possibilities for $G$. This completes the proof.

\section{REFERENCES}

1. R. D. M. Accola, On the number of automorphisms of a closed Riemann surface, Trans. Amer. Math. Soc. 131 (1968), 398-408. MR 36 \#5333.

2. L. Greenberg, Note on normal subgroups of the modular group, Proc. Amer. Math. Soc. 17 (1966), 1195-1198. MR 33 \#7423.

3. M. Newman, Classification of normal subgroups of the modular group, Trans. Amer. Math. Soc. 126 (1967), 267-277. MR 34 \#4217.

4. H. Zassenhaus, Lehrbuch der Gruppentheorie, Teubner, Leipzig, 1937; English transl., Chelsea, New York, 1949. MR 11, 77.

National Bureau of Standards, Mathemat ics Division, Washington, D.C. 20234 